\title{
Quo vadis antiviral agents for herpes, influenza and HIV?
}

The discovery of the nucleoside analogue acyclovir and the establishment of its biochemical mode of action as an anti-herpes agent were the scientific foundation stones for medical investigations which have proved its wide clinical usefulness over the last two decades. I remember clearly the initial launch of the drug in London and it was with considerable interest that I attended recent meetings to discuss the antiviral activity of "sisters" of acyclovir, namely, famciclovir, penciclovir and, later vancyclovir ${ }^{1.2}$ It did not appear likely in the 1970 s that a nucleoside analogue, or at least the triphosphate derivative, could have such a narrow specificity against a viral enzyme -namely herpes DNA polymerase-without inhibiting cellular DNA polymerases. Countless scientific investigations have since demonstrated the relative safety of the compound, ${ }^{3}$ its antiviral spectrum, its ability to prevent viral spread and recurrent infection, and to alleviate the symptoms following tissue destruction by the virus. Drug-resistant herpes viruses have been slow to appear. ${ }^{4}$

Given the success of acyclovir do we need alternative anti-herpes compounds or could the efficacy or even the antiviral range of acyclovir be improved? The answer to the first question must be "yes". New antiviral agents are always required, if for no other reason that to treat persons infected with drugresistant viruses, who include immunosuppressed patients such as those receiving transplants or those infected with HIV. The antiviral agent foscarnet has a mode of action completely different from that of acyclovir and, as expected, cross-resistance is not a major problem. The drug is used to treat immunosuppressed patients suffering from serious infections caused by acyclovir-resistant herpes viruses and CMV.

A narrow antiviral spectrum has been a problem with every antiviral agent. Amantadine inhibits only influenza $A$ virus replication and not influenza $B$ virus; zidovudine inhibits only HIV-1; acyclovir may be very potent against HSV-1 but is much less so against VZV. Could this problem be circumvented by increased dosages of acyclovir or, alternatively, by discovering closely related analogues which may produce high plasma levels in the body because of increased half-life or different dosage scheduling? The task is to discover compounds which are similar enough to acyclovir in molecular structure to give confidence that they will have the same safety profile, but different enough to have new antiviral properties and to be patentable. Ganciclovir has a different molecular structure, a different antiviral spectrum to include CMV but also, unfortunately, a much less favourable toxicological profile. $^{5}$ SmithKline Beecham chemists synthesised famciclovir and penciclovir ${ }^{6-8}$ whilst Burroughs Wellcome have produced valaciclovir. ${ }^{9}$ Famciclovir is a pro-drug which is de-acetylated and oxidised to penciclovir giving high bioavailability of the latter molecule. The half-life is somewhat prolonged and so dosages may be wider apart. Similarly valaciclovir, the L-valyl ester of acyclovir, is hydrolysed to acyclovir giving, after oral administration, plasma concentrations equivalent to those obtained after intravenous acyclovir. At present all three appear to be hopeful antiviral agents with a useful future.

Can we draw from the herpes success some meaningful conclusions to help us with two more intractable viral candidates for extinction or, at least, containment: influenza A and HIV-1? The chemists' favourite molecule, the nucleoside analogue, still has much potential, but the incredible mutation rates of RNA viruses is an inherent problem. Ho et al. ${ }^{10}$ have described the emergence of drug-resistant mutants within days of onset of antiviral chemotherapy of AIDS patients with a protease inhibitor, but the same study also described the milieu in which some antiviral compounds exist; this has been described as a "mean street" with billions of virions being eradicated daily by millions of activated $\mathrm{T}$ cells. ${ }^{11}$ At first sight, the situation may appear completely hopeless for any but the most powerful inhibitor. Although herpes viruses also undergo massive replication in the body and can be stopped by antiviral agents, as demonstrated clearly with acyclovir, DNA viruses do not have the propensity for the high mutation rates of RNA viruses and so constitute easier targets. A trip back in time to the ice age would be required to discover as many herpes mutants as one would discover with influenza or HIV-1 in a journey lasting a single year. Nevertheless there is no overwhelming evidence that a patient would succumb to an infection with drugresistant HIV or, indeed, a drug-resistant influenza $\mathrm{A}$ virus. Rather, at least with HIV, mixed populations of pro-viral genomes exist in a patient treated with zidovudine and even after long periods of drug treatment, ${ }^{12}$ zidovudine-sensitive genome sequences can still be detected. ${ }^{13}$

Amantadine has been shown to exert a strong and reproducible antiviral effect when used as a prophylactic agent against human sub/types of influenza $\mathrm{A}$ virus including $\mathrm{H} 2 \mathrm{~N} 2, \mathrm{H} 1 \mathrm{~N} 1$ and H3N2. ${ }^{14}$ Drugresistant viruses can be selected in the laboratory ${ }^{15}$ and in the patient ${ }^{16}$ and yet treated patients continue to benefit from therapy. Amantadine- or rimantadineresistant strains of influenza A were isolated even 
before the drug was used ${ }^{17}$ whilst viruses resistant to zidovudine or non-nucleoside inhibitors of HIV can be isolated from a minority of patients before drug therapy is commenced. ${ }^{13}$ Pre-existing polymorphisms exist in the particular nucleotides of HIV reverse transcriptase or protease coding for amino-acid substitution which, in the corresponding viral protein, would result in a configuration change and drug resistance.

Given these achievements, where will antiviral agents proceed in the next few years? Inevitably, the solution of further X-ray structures of viral proteins and computerised imaging will be keys to new developments. Eleven years ago a group of scientists in Melbourne published the first data on the atomic Xray structure of influenza $A$ virus neuraminidase. This enzyme is not unique to viruses; similar ones are widespread in the animal kingdom. These enzymes are glycohydrolases which cleave terminal sialic acid from glycoproteins, glycolipids or oligosaccharides. In virological terms the enzyme functions at the stage of influenza virus release from an infected cell. The main study of anti-neuraminidase drugs commenced a quarter of a century ago with the synthesis of the Neu5Ac2en, a derivative of sialic acid which inhibited viral, bacterial and mammalian sialidases. A series of these compounds was tested as anti-influenza virus drugs $^{18}$ and although a number of analogues of Neu5Ac2en inhibited influenza virus replication in cell culture, no antiviral activity was detected in animal models. In retrospect, this negative finding may be attributed to the rapid metabolism of the early compounds tested; newly synthesised analogues produce a specific anti-influenza effect in vivo ${ }^{19}$ and clinically, ${ }^{20}$ after topical application.

Influenza sialidase is a tetramer of identical subunits. There are six four-stranded anti-parallel $\beta$-sheets arranged as if in the blades of a propeller. The active enzyme site is a deep cavity on the neuraminidase protein surface and is lined entirely by amino acids that are invariant in sialidases of all strains of influenza $A$ and $B$ which have been characterised. In contrast, variable amino acids are found next to and encircling the active site and these are antigenic determinants reacting with post-infection anti-neuraminidase antibodies. The scientific trick is to avoid inhibitors binding to these variable amino acids but rather to select molecules to the invariant amino acids.

Sialic acid, the product of the enzyme-catalysed reaction, binds to the active site of the enzyme in a "boat" configuration. The carboxylate interacts with three invariant arginine residues on the neuraminidase enzyme. In this conformation it resembles the geometry of the potent sialidase inhibitor (Neu5Ac2en), as noted above. Importantly, strain invariance extends to a number of other amino acids which do not themselves make contact with sialic acid, but which provide a scaffold on which amino acids contacting the bound sugar are supported.

The Melbourne group made predictions from X- ray-derived structures of energetically favourable substitutions to the unsaturated sialic acid analogue Neu5Ac2en. The most interesting of these chemical modifications was the replacement of the hydroxyl group at the four position on Neu5Ac2en by an amino group. Substitutions of the 4-hydroxyl group by an amino group should produce a significant increase in the overall binding interaction due to a salt bridge formation with the side chain carboxylic acid group of Glu 119 in the enzyme. Similarly, the replacement of the same hydroxyl groups by the significantly more basic guanidine group was predicted to produce an even tighter affinity of the substituted Neu5Ac2en for the active site as a result of lateral binding through the terminal nitrogens of the guanidine group with both Glu 119 and Glu 227. Direct measurements of viral neuraminidase enzyme inhibition showed that the 4amino and 4-guanidino-substituted Neu5Ac2en were, indeed, high-affinity inhibitors for the influenza virus enzyme. Moreover, the compounds inhibited influenza virus plaque formation in cell culture. Most excitingly, the compounds had antiviral effects in vivo: Hayden et al. ${ }^{20}$ using intranasal prophylaxis, were unable to recover virus from 16 drug-treated volunteers, whereas virus was recovered from $65 \%$ of 17 untreated controls. The compound looks most interesting.

But I have an important reservation about current searches for inhibitors of HIV. With influenza infections of the respiratory tract there is clear evidence of viral replication in the trachea, bronchi and alveoli. The disease is clearly related to known pathological changes and to viral replication, and inhibitors of viral replication have a logical scientific basis for clinical action. This has been shown with amantadine and with the anti-neuraminidase compound. Similarly, herpes viruses cause cellular destruction in the skin, eye or brain and these pathological changes are stopped by antiviral compounds. But is the pathology of HIV so clear cut? Ho et al. ${ }^{10}$ clearly believe it is. In their important study the antiviral effects of ABT 538, an inhibitor of HIV protease, were quantified by the reduction in viral genomes and, in half the patients, by an increase in CD4 cell count. In approximately onethird of the treated patients, no increase in CD4 cell count was noted, whilst the remaining patients formed an intermediate group. Thus uncertainty remains as to whether viral replication per se is destroying CD4 cells. What if the exquisitely hostile environment created by the immune system in AIDS patients ${ }^{11}$ is also directed to self:? Habeshaw et al. ${ }^{21}$ have steadfastly maintained that a strong auto-immune component can be recognised in AIDS patients by virtue of stimulation of subsets of autoreactive T cells. Supporters of the hypothesis point to activation of CD4 lymphocytes as a key feature of the pathogenesis. There is evidence that these $\mathrm{T}$ cell subsets kill uninfected CD4 cells as well as virus-infected cells. These autoreactive $\mathrm{T}$ cells, which are normally suppressed, recognise in an activated $\mathrm{T}$ cell a portion of the $\mathrm{C}$ terminus of $\mathrm{gp} 120$, which when presented on a class I molecule mimics the 
normal presentation of an allo-epitope HLA class II $\beta$ chain. ${ }^{22}$ If auto-immunity contributes significantly to the pathogenesis of AIDS then new vistas appear for chemotherapists. Certainly new inhibitors of viral replication will still be the cornerstone of treatment but would need to be used as soon as the patient is infected to reduce the opportunity of recognition of class II $\beta$ chain presented on HLA class 1 by these preexisting autoreactive $T$ cells. Later possibilities for chemotherapy would be drugs to mask the crossreactive allo-epitope on the $C$ terminus of the gp 160

\section{References}

1. Field HJ. Famciclovir/penciclovir: Pharmacology and Pharmacokinetics Workshop. Proceedings of a meeting held at Cliveden, Maidenhead, UK, on 23 April 1993. Antiviral Chem Chemother 1993; 4 (Suppl. 1).

2. Field HJ, Griffiths PD, Oxford JS. Evaluating new antiviral drugs. Proceedings of a meeting held in Switzerland, 17-20 December, 1993. Antiviral Chem Chemother 1994;5 (Suppl. 1).

3. Tilson BB, Eagle CR, Andrews EB. Safety of acyclovir: a summary of the first 10 years experience. $J$ Med Virol 1993; (Suppl. 1): 67-73.

4. Collins $P$, Ellis $M N$. Sensitivity monitoring of clinical isolates of herpes simplex virus to acyclovir. J Med Virol 1992; (Suppl. 1): $58-66$.

5. Safrin S, Crumpacker C. Chatis $\mathrm{P}$ et al. A controlled trial comparing foscarnet with vidarabine for acyclovir-resistant mucocutaneous herpes simplex in the acquired immunodeficiency syndrome. $N$ Eng $J$ Med 1991: 325: $551-555$.

6. Boyd MR, Safrin S, Kera ER. Penciclovir: a review of its spectrum of activity, selectivity, and cross-resistance pattern. Antiviral Chem Chemother 1993; 4 (Suppl. 1): 3-11.

7. Harnden MR, Jarvest RL. An improved synthesis of the antiviral acyclonucleoside 9-(4-hydroxy-3-hydroxymethylbut-1-yl) guanine Tetrahedron Lett 1985; 26: 4265-4268.

8. Vere-Hodge RA, Sutton D, Boyd MR, Harnden MR, Jarvest RL. Selection of an oral pro-drug (BRL 42810 Famciclovir) for the antiherpes virus agent BRL 39123 [9-(4-hydroxy-3hydroxymethylbut-1-yl) guanine; penciclovir. Antimicrob Agents Chemother 1989; 33: 1765-1773.

9. Weller S, Blum MR, Doucette M et al. Pharmacokinetics of the acyclovir pro-drug, valaciclovir, after escalating singleand multiple-dose administration to normal volunteers Clin Pharmaco Therapeut 1993 54: 595-605.

10. Ho DD, Neumann AV, Perelson AS. Chen W, Leonard JM, Markowitz M. Rapid turnover of plasma virions and CD4 lymphocytes in HIV-1 infection. Nature 1995; 373: 123-126. viral spike or, more controversially, the use of selective immunosuppressive drugs to counteract the autoimmune reaction. But perhaps most exciting would be the novel possibility of tolerising an AIDS patient with high concentrations of the mimic peptide or by transfusing with antibodies to the mimic peptide.

J. S. OXFORD

Department of Medical Microbiology, The London Hospital Medical College, Turner Street, London E1 2AD

11. Wain-Hobson S. Virological mayhem. Nature 1995; 373: 102.

12. Stein CA, Levantis P, Goh B, Hillman R, Oxford JS. Limiting dilution PCR to detect changes in the RT of patients on long term AZT treatment. Tenth International Congress on AIDS. Yokohama, Japan, 1994.

13. Stein CA, Levantis P, Oxford JS. Sequence analysis of proviral HIV RT amplified directly by a semi-quantitative technique from AZT treated patients. J Med Virol 1994; 44: 115-121.

14. Oxford JS. Chemotherapy of influenza and respiratory viruses. In: Dimmock NJ, Griffiths PD, Madeley CR (eds) Control of virus diseases. Society for General Microbiology, Symposium no. 45. Cambridge, Cambridge University Press 1990, pp. 213-242.

15. Oxford JS, Logan IS, Potter CW. In vivo selection of an influenza A2 strain resistant to amantadine. Nature 1970; 226: $82-83$

16. Hayden FG, Hay AJ. Emergence and transmission of influenza A viruses resistant to amantadine and rimantadine. Curr Topics Microbiol Immunol 1992; 176: 119-130.

17. Heider H, Adamczyk B, Presber HW, Schroeder C, Feldblum $R$, Indulen MK. Occurrence of amantadine- and rimantadine-resistant influenza A strains during the 1980 epidemic. Acta Virologica 1981; 25: 395-400.

18. Palese P, Schulman JL. Inhibitors of viral neuraminidase as potential antiviral drugs. In: Oxford JS (ed) Chemoprophylaxis and virus infections of the respiratory tract, vol 1. Cleveland, CH, CRC Press, 1977.

19. Von Itzstein $\mathbf{M}, \mathbf{W u} \mathbf{W}-\mathrm{Y}$, Kotz GB et al. Rational design of potent sialidase-based inhibitors of influenza virus replication. Nature 1993; 363: 418-423.

20. Hayden FG, Lobo M, Esinhart J, Hussey E. 34th Interscience Conference on Antimicrobial Agents and Chemotherapy, 1994.

21. Habeshaw JW. HLA mimicry by HIV-1 gp 120 in the pathogenesis of AIDS. Immunol Today 1994; 15: 39-40.

22. Wilson SE, Hounsell E, Habeshaw JA. Generation of auto and allo cytotoxic $\mathrm{T}$ cell responses by regions of HIV gp 120 which share structural homology with human MHC class I/II molecules. IXth International Conference on AIDS. Berlin, 1993. 